\title{
Arquitectura institucional, contexto sociocultural e integridad electoral ${ }^{\star}$
}

\author{
DiETER NOHLEN*
}

\begin{abstract}
Para citar este artículo: Nohlen, D. (2016). Arquitectura institucional, contexto sociocultural e integridad electoral. Desafíos, 28(I), 429-453.
\end{abstract}

Voy a disertar sobre arquitectura institucional, contexto sociocultural e integridad electoral, y precisamente, sobre la compleja interrelación de los tres fenómenos. Trataré el tema de la arquitectura institucional y de la integridad electoral en un nivel algo abstracto, que en el título de esta primera sesión del Segundo Congreso de la AWEB está puesto en una relación estrecha y específica -"la institucionalidad electoral necesaria para garantizar la integridad electoral". No voy a referir ni comentar los manuales de práctica y justicia electoral que han aparecido en el último decenio y que informan sobre estándares internacionales de institucionalidad electoral, manuales de consulta, dirigidos a los órganos electorales y los observadores electorales, dado que contienen valiosas instrucciones sobre cómo organizar elecciones libres y honestas y cómo evaluar su calidad democrática. ${ }^{1}$ Expondré consideraciones más bien conceptuales, conforme a lo que enfatizaba

\footnotetext{
* Texto revisado y ampliado de la conferencia ofrecida en la Segunda Asamblea General de la Association of World Elections Bodies (AWEB), Punta Cana, República Dominicana, en la primera sesión del pleno: Arquitectura electoral: la institucionalidad necesaria para garantizar la integridad electoral, 20 de Agosto de 2015. Revisión lingüística del Dr. José Reynoso Núñez, Guadalajara.

** Dr. phil., Dr. h.c. mult., profesor emérito de Ciencia Política de la Universidad de Heidelberg, Alemania. Correo electrónico: dieter.nohlen@urz.uni-heidelberg.de

1 Véanse entre otras publicaciones: United Nations, (2005); United Nations Organization y European Commission for Democracy Through Law (Venice Commission) (2006); Council of Europe y OEA, (2008) Merloe, P. (2008); The Carter Center, (2013); IDEA Internacional, Tribunal Electoral, UNAM (2013).
} 
Max Weber, que sin conceptos no se obtiene conocimiento alguno y "no las conexiones 'de hecho' entre 'cosas' sino las conexiones conceptuales entre problemas están en la base de la labor de las ciencias" (2001, p. 57). Tomaré a veces como punto de referencia el Proyecto de Integridad Electoral de Pippa Norris y colaboradores, ${ }^{2}$ uno de los proyectos más ambiciosos en ciencias sociales para medir la integridad electoral a nivel mundial. Incluiré en mis consideraciones el contexto sociocultural en el que se celebran elecciones. El contexto constituye el marco histórico para reflexionar sobre las instituciones, sus funciones y sus efectos reales. Me voy a referir al contexto latinoamericano, a veces contrarrestándolo con el de otras regiones del mundo, a partir de mis propias investigaciones y de mis experiencias como observador participante. $^{3} \mathrm{Al}$ tomar en cuenta el contexto, integro la empíria en mis consideraciones, más aún, la relación con el contexto va a poner en

2 El Proyecto de Integridad Electoral se propone el estudio de las elecciones en todo el mundo con un enfoque comparativo, centrándose, como expresa su auto-definición, en tres cuestiones: ¿Qué se necesita para que unas elecciones correspondan a estándares internacionales? ¿Qué pasa cuando las elecciones no consiguen cumplir con ellos? ¿Qué se puede hacer para mitigar estos problemas? La "Percepción de Integridad Electoral" (en siglas inglesas PEI) representa la parte empírica-cuantitativa del proyecto. Como objetivo del PEI autoproclama la presentación de "una comprensiva, imparcial e independiente fuente de información, derivada de evaluaciones de expertos en relación a si las elecciones corresponden a principios y estándares internacionalmente reconocidos" El proyecto tiene su base en la Universidad de Sydney y en la Harvard's Kennedy School of Government. Su directora es Pippa Norris. Se han publicado ya varios estudios que explican el diseño de investigación comparativa e informan sobre primeros resultados: Norris, P. (2013); Norris, P., Martínez i Coma, F. y Frank, R.W. (2013); Norris, P., Frank, R.W. \& Martínez i Coma, F. (Eds.); Norris, P. (2014); Norris, P., Frank, R. W. \& Martínez i Coma, F. (Eds.) 2014; Norris, P., Frank, R.W. \& Martínez i Coma, F. (Eds.) (2015). Agradezco a Ferran Martínez i Coma el comentario crítico a una versión anterior de este ensayo.

3 Al respecto: Sternberger, D., \& Vogel, B. (Eds.), Nohlen, D. (Red.) (1969); Nohlen, D. (1981); Jaramillo, J., León-Roesch, M., \& Nohlen, D. (1989); Nohlen, D., Picado, S., \& Zovatto, D. (Comps.) (1998); Nohlen, D. (Ed.) (1993); Nohlen, D. (2004); Nohlen, D. (Ed.) (2005); Nohlen, D., Zovatto, D., Orozco, J., \& Thompson, J. (Comps.) (2007); Nohlen, D. (2008); Ortiz, R.(Ed.) (2009); Nohlen, D. (2015a). Sobre otros continentes véanse Nohlen, D. (2014a); Nohlen, D., \& Kasavović., M. (1996); Nohlen, D., Krennerich, M., \& Thibaut, B. (Eds.) (1999); Nohlen, D., Grotz, F., \& Hartmann, C. (Eds.) (2001); Nohlen, D., \& Stöver, P. (Eds.) (2010). El proyecto de integridad electoral no parece conocer ninguno de estos trabajos así como tampoco estudios escritos en castellano, por ejemplo, toda la valiosa labor, de 1989 en adelante, del Instituto Interamericano de Derechos Humanos y su Centro de Asesoría y Promoción Electoral con sede en San José de Costa Rica. 
cuestión el supuesto de esta primera sesión del Congreso consistente en que se pueda determinar en términos generales una necesaria institucionalidad para garantizar la integridad electoral. Este objetivo deseado depende de varios factores, no sólo institucionales, sino también de aquellos factores que integro en el concepto de "contexto sociocultural". Este se expresa ya en puntualizar que el grado de dependencia de los factores institucionales y no institucionales varía según lugar y tiempo, incluso de una elección a otra. Por esta compleja interrelación, el posible alcance de la arquitectura institucional para la integridad electoral depende del contexto, por ejemplo del tipo de régimen, conforme a mi tesis: el contexto hace la diferencia (Nohlen, 2003). ${ }^{4}$ De antemano, quisiera enfatizar que en mi opinión, las instituciones importan mucho, pero de forma relativa. En concreto, la necesaria arquitectura institucional es aquella que se adecua al contexto, un diseño institucional, administrativo y jurisdiccional que corresponde a los desafíos concretos y variantes de organizar unas elecciones libres y honestas. Mucha incidencia, sin embargo, acoge la manera como se definen los fenómenos que se interrelacionan.

\section{Consideraciones preliminares sobre el concepto de integridad electoral}

Esto es especialmente válido para el concepto de integridad electoral. que, en su reciente expansión que empieza a sustituir en América Latina al de la justicia electoral en su sentido amplio. ${ }^{5}$ Este nuevo concepto es clave en lo que se estudia aquí, porque es el firme objetivo normativo y práctico, en contraste con medios y circunstancias aquí relacionados con ello, que pueden variar y que se observan en relación con ella.

Varias cuestiones me guían. En primer lugar, ¿es la integridad electoral un concepto genérico, considerado para unificar conceptos y

\footnotetext{
4 Ver también Nohlen, D. (2006) y Nohlen, D. (2013).

5 Ver Orozco Henríquez, J. (Comp.) (1999a); Orozco Henríquez, J. (Comp.) (1999); Nohlen, D. (2004); Nohlen, D. (2010); Ortiz Ortiz, R. (Ed.) (2009); Nohlen, D. (2015).
} 
fenómenos dispersos interrelacionados entre ellos, o un concepto entre otros en el área electoral para garantizar elecciones libres y honestas, como, por ejemplo, independencia, imparcialidad, transparencia, profesionalismo y dedicación al servicio (service-mindedness), fundamental guiding principles destacados por parte de IDEA Internacional? (Leterme, 2015). ${ }^{6}$

En segundo lugar, ¿es la integridad electoral un fenómeno objetivo, comprobable por el examen de la correspondencia entre práctica electoral y el concepto normativo mismo? O es, al mismo tiempo, un fenómeno subjetivo, o sea, expuesto a su percepción por parte de individuos y colectivos que o participan en las elecciones mismas o lo observan y evalúan? Es una doble perspectiva, parecida a la conocida del concepto de legitimidad: legitimidad atribuida a instituciones por la legitimidad de validez de su diseño, con el que se garantiza la validez de algunos principios fundamentales, o por la legitimidad de creencia, el reconocimiento de las instituciones de ser legítimas por parte de los miembros de una sociedad. ${ }^{7}$ En ambos conceptos se distinguen entre validez intrínseca, inherente, y validez extrínseca, de creencia. A esta disyuntiva volveré más tarde. Aquí me restrinjo a decir que en el debate sobre calidad electoral se observa un uso poco lógico de ambos conceptos y especialmente de su interrelación.

En tercer lugar, ¿'es integridad electoral un concepto estrecho o amplio, limitado a algunas características o abierto a características que se ofrecen según lugar y tiempo? La pregunta alude al problema de la supra-extensión de un concepto (conceptual stretching), causada por la expansión de las dimensiones características de éste con el efecto de una excesiva diferenciación interna. ${ }^{8} \mathrm{~A}$ la hora de desarrollar un concepto, frecuentemente se ven integradas en éste cualidades que, bien mirado, podrían ser entendidas como variables susceptibles de

\footnotetext{
6 Ver Global Commission on Elections, Democracy and Security (2012) ACE (The Electoral Knowledge Network) (2012); Cordenillo, R., \& Ellis, A. (Eds.) (2012).

7 Ver Weber, M. (1974); Easton, D. (1979); Sternberger, D.(1967); Bobbio, N. (1981); Nohlen, D. (2015b).

8 Véase al respecto Sartori, G. (1991) Nohlen, D. (2006) y Nohlen, D. (2013).
} 
ser investigadas en su relación mutua. La inclusión, por ejemplo, de la participación electoral en el concepto de integridad electoral, entendida como integración del ciudadano en el proceso electoral, es un evidente caso de supra-extensión conceptual. Conduce a suponer, que un bajo grado de participación electoral expresa una falla de integridad electoral. Sin embargo, no conviene cargar a la integridad electoral con un fenómeno que, de verdad, solo puede percibirse en relación con ella, una relación que llama a que sea investigado. En este sentido, fallas en integridad electoral pueden tener efectos de mayor y menor participación electoral. Diferenciando aquí sólo entre dos factores, la desconfianza en el proceso electoral tiende a disminuir la participación electoral, el clientelismo electoral, a aumentarla. ${ }^{9} \mathrm{Fa}-$ llas de concepualización y diferenciación pueden producir enormes errores de análisis. Por cierto, la participación electoral depende de múltiples factores. Además, no conviene sobreestimar el factor integridad electoral, pues desde una perspectiva comparativa, países de comprobada integridad electoral pueden variar mucho en cuanto a la participación electoral. Tampoco conviene suponer que países con baja participación electoral tengan problemas de integridad electoral. Por más importante y más equitativa que sea, la participación electoral es sólo un mecanismo en el que se expresa la participación política.

\section{El concepto de integridad electoral hacia dentro y hacia afuera}

Ahora bien: Respecto a lo que se puede entender por integridad electoral, voy a diferenciar dos dimensiones. La primera se refiere a su alcance integrador, es decir "asume una visión integral de todas las dimensiones del ciclo electoral partiendo del diseño de la legislación y de los organismos y la selección de autoridades electorales, hasta los procesos de votación, conteo, divulgación de resultados y fiscalización."(Centro de Estudios Políticos, 2014). Esta dimensión se fija en lo que se integra, tiende a que su alcance sea completo: el

\footnotetext{
Vér Birch, S. (2010); Carreras, M. \& Irepoglu, Y. (2013); Carreras, M. \& Posvar Hall, W. (2014); Nikolayenko, O. (2015).
} 
sinónimo de tal aspiración es la totalidad. ${ }^{10}$ Integridad electoral, en esta su primera dimensión, se asocia con la idea de enfocar un ciclo electoral con diferentes etapas. Aunque esta perspectiva no es tan novedosa, como supone (Nohlen, Picado \& Zovatto, 1998), enfatiza la importancia de atender las etapas anteriores y posteriores a la jornada electoral en el análisis de las elecciones -hoy en día por la alta incidencia del financiamiento de los partidos y de las campañas electorales, así como por el acceso a los medios de comunicación en la contienda electoral- sin duda clave en la evaluación de las elecciones con criterios democráticos. ${ }^{11}$

Integridad electoral, en su segunda dimensión, sin embargo, se refiere a la calidad de las elecciones, precisamente a la relación entre valores y prácticas. Postula la correspondencia entre valores y normas por un lado y comportamientos y resultados por el otro: el sinónimo de tal aspiración es la honestidad. Integridad electoral se determina como el postulado ético, dirigido al proceso electoral en total, a los individuos involucrados en él, para comportarse de forma íntegra, o sea honesta, conforme a los valores y las normas que sustentan elecciones democráticas, y para proteger, en su caso, la honestidad del proceso electoral frente a desafíos que la ponen en cuestión.

Es importante señalar que el Proyecto de Integridad Electoral de Pippa Norris y colaboradores confiesa ambos sentidos de integridad electoral, aunque permite que confluyan en el concepto más elementos de diferente índole (Pippa Norris, P., Elklit, J., \& Reynolds, A. 2015). Además, el proyecto determina bien los valores que gobiernan la integridad electoral, manifestados en las normas internacionales (Norris, 2014). Son los acuerdos multilaterales, convenciones, tratados y protocolos internacionales que se refieren a los derechos humanos y

\footnotetext{
10 Pero ojo, la idea de la totalidad seduce a incluir en el concepto fenómenos más allá de lo que conviene integrar y a descuidar necesarias diferenciaciones. Conduce, además, a diseñar una imagen completa, condensada tal vez en una única cifra, en vez de indagar bien la constelación histórica-específica del caso en su lugar y tiempo.

11 La crítica a la observación electoral se fija sobre todo en que ella se restringe precisamente a la jornada electoral (Kelley, 2010, p. 163).
} 
a los derechos políticos, de donde se desprenden las elecciones como los mecanismos necesarios de participación de los ciudadanos en la formación de la voluntad política. ${ }^{12}$ Con este trasfondo de valores y normas, se puede, entonces, evaluar la integridad electoral ex negativo. Si no se lesionan las normas, si no se manipulan elementos del proceso electoral en contra de lo legalmente o constitucionalmente establecido, y en última estancia, si no se contradice, más allá de las normas, a los valores democráticos que deben sustentarlas, entonces existe integridad electoral.

En su ausencia, a menudo se habla de malas prácticas, distinguiendo sólo entre sus diferentes grados. Sin embargo, conviene diferenciar entre irregularidades electorales y fraude o manipulación electoral, ${ }^{13}$ una importante distinción, pues irregularidades en el sentido de errores pueden ocurrir en cualquier momento, ya que son consustanciales al ser humano. En este sentido, integridad electoral no puede significar ausencia de errores, aunque las faltas generalizadas pueden tener graves consecuencias respecto a la confianza en los resultados electorales, en especial, cuando los medios de comunicación les magnifican indebidamente, las confunden para este efecto con fraude o

12 Así, la Carta Democrática Interamericana, aprobada el 11 de septiembre de 2001, dispone en su artículo 3: "Son elementos esenciales de la democracia representativa, entre otros, el respeto a los derechos humanos y las libertades fundamentales; el acceso al poder y su ejercicio con sujeción al estado de derecho; la celebración de elecciones periódicas, libres, justas y basadas en el sufragio universal y secreto como expresión de la soberanía del pueblo, el régimen plural de partidos y organizaciones políticas; y la separación e independencia de los poderes públicos". Respecto a observación e integridad electoral, véase Lusverti, C. F. (2014).

13 Contrario a la gran mayoría de los estudios, entre ellos los realizados por Carreras e Irepoglu (2013), que no diferencian entre irregularidad y manipulación. Esta negación es inherente al enfoque de la reciente corriente Forensics que trata de detectar el fraude electoral por medio de un tratamiento estadístico de datos (véanse, por ejemplo, Beber \& Scacco (2012) y Leemann \& Bochsler (2014). Estos análisis de nuevo se limitan al día de la elección, a la entrega y al conteo de los votos, comparando estos datos con otros de orígen diferente (datos registrales, encuestas preelectorales, exitpoll data, etc.), que a veces son menos confiables que los propios resultados electorales oficiales. En vez de contar con la falibilidad del ser humno y la posibilidad de errores involuntarios, el enfoque supone la regular omnipresencia del fraude electoral. Ya el propio método digital no permite distinguir entre irregularidades y fraude. Por supuesto, esta diferenciación hace necesario un fino enfoque cualitativo, que contrasta con el mainstream cuantitativo. 
manipulación, y los perdedores de la contienda aprovechan la confusión para tratar de deslegitimar el resultado electoral. El fraude o manipulación electoral, en contraste, tiene que ser percibido como actitud intencionada, en contra de la integridad electoral. Produce desconfianza y reduce la legitimidad de las elecciones y su resultado. Puede traer severas consecuencias. Atañe incluso el tipo de régimen, aunque los estudios de integridad electoral, específicamente el índice de Perception of Electoral Integrity (PEI) abordan la cuestión de su vigencia universalmente en todos los países sin diferenciar entre tipos de regímenes.

\section{El concepto de arquitectura institucional}

Respecto a la arquitectura institucional, en un principio, parece claro lo que se entiende por este concepto en el ámbito electoral: órganos electorales, normas, convenciones y formas de interacción que regulan el proceso electoral. Las teorías institucionales sostienen que las instituciones pueden explicar comportamientos y resultados políticos. De allí el supuesto de que las instituciones garantizan la integridad electoral y que hay que encontrar e introducir estas instituciones que inducen tales efectos. El nuevo institucionalismo, sin embargo, pone en cuestión la existencia de una relación determinística entre arquitectura institucional e integridad electoral. Como ya decía, la arquitectura institucional tiene importancia, pero ésta es relativa. Mi postura institucional-contextual no desvalora las instituciones, sino reconoce, precisamente, que la administración electoral puede jugar un rol importante, como lo ha sostenido por ejemplo Robert A. Pastor (1999), considerándola incluso como "una variable clave para explicar el éxito o el fracaso de una transición democrática". ${ }^{14}$ Esta relevancia del objeto ha motivado mi propia dedicación a los órganos electorales y al proceso electoral. En tiempos autoritarios, la

\footnotetext{
14 Es cierto que cuentan muchos detalles, lo que tiene que tenerse presente, aunque el discurso se lleva a cabo en un nivel abstracto. Por ejemplo, importa el diseño de las boletas, el orden en el que los partidos y candidatos aparecen, impacta en las cruces que los votantes colocan en ellas. Para profundizar al respecto consultar Darcy, R., \& McAllister, I. (1990), y Koppel, J. G.S., \& Stehen, J. A. (2004).
} 
oposición pide elecciones libres, y a veces, establecer las condiciones legales y administrativas para la celebración de elecciones libres puede producir un cambio de régimen. Con su reforma de la administración y judicialización electorales de 1996, que pude presenciar de cerca, México es tal vez el caso más paradigmático de tal desarrollo exitoso. Pero los efectos que puedan tener las instituciones dependen en gran medida de otros factores como señalan sobre todo los casos de transición o consolidación democráticas frustradas, por ejemplo en los países de la "primavera árabe" (ver Nohlen, D. 2010).

Tomando en cuenta la doble dimensión del concepto de integridad electoral, se puede sostener que existen ideas de lo que debe incluir una necesaria arquitectura electoral. Pues el propio concepto integrador de integridad electoral postula, en términos generales, órganos y procedimientos electorales necesarios para cubrir todo el proceso electoral. Cumplido el aspecto de totalidad del concepto, la cuestión debe dirigirse hacia el tipo o la estructura de la arquitectura institucional. La experiencia internacional comparativa señala que no existe ningún modelo de arquitectura ideal y que hay alternativas de diseño institucional, por ejemplo en relación al lugar y status de los órganos electorales en la estructura estatal, autónomos e independientes o parte integrante del poder judicial, a la organización concentrada o desconcentrada del "poder electoral", a la adjudicación de facultades (administrativas, registrales, jurisdiccionales) en caso de desconcentración y descentralización de los órganos electorales, etc. ${ }^{15}$ Además, fijándose en la arquitectura institucional y en las normas formales, seduce a limitarse en el análisis a las grandes confesiones de fe y descuidar la práctica electoral, por ejemplo, descuidar el hecho de que bastantes veces no existen sanciones para el caso de no cumplir con las normas legales presentadas como supuestos indicadores de integridad electoral.

El parámetro de la evaluación de integridad electoral es el resultado (outcome) de la honestidad, consideración que reintegra la segunda dimensión de su concepto al análisis, la correspondencia de la práctica

15 Para profundizar en este tema consultar Jaramillo, J. (2007). 
con valores y normas que se deducen de la teoría de la democracia y de los derechos humanos. Se puede distinguir, sin embargo, entre situaciones, en las que ya la arquitectura institucional formalizada falla (por ejemplo cuando los distritos electorales se establecen de forma políticamente sesgada), ${ }^{16}$ y aquellas, en las que el comportamiento de los individuos y colectivos involucrados en el proceso electoral, la hace fallar, aunque se ha invertido una parte considerable del producto nacional en el establecimiento de una arquitectura institucional permanente y se sigue gastando mucho en su mantenimiento. La arquitectura institucional misma no genera ni garantiza honestidad en su manejo. Por otra parte, se observa a menudo que la misma institucionalidad electoral se asocia con honestidad en este y falla en otro lugar, y que hay resultados de honestidad parecidos, aunque la institucionalidad formal sea distinta. En este sentido, convence que el índice de Perception of Electoral Integrity (PEI) no se fija en las estructuras institucionales mismas, sino en el grado respectivo de cumplimiento de cada una de ellas con los requisitos de una elección democrática. La honestidad en el manejo de lo electoral es el criterio clave.

En dos oportunidades (entre 49 ítems, en total), el índice PEI recurre, sin embargo, a características de tipo institucional. Ambas exhiben una enorme importancia porque con ellas se alude al régimen político, cuando se pregunta por la vigencia de la ley y la imparcialidad de los órganos electorales. Como se sabe, en regímenes autoritarios, sin separación de poderes ni vigencia del Estado de derecho, la ley es un arma del poder contra la oposición, y la correspondencia con la ley en términos de un positivismo jurídico no garantiza la integridad electoral. En el autoritarismo, tampoco se garantiza la independencia y la autonomía de los órganos electorales.

\footnotetext{
16 El sistema electoral, o sea la forma en que la ciudadanía vota y el mecanismo por medio del cual los votos se convierten en escaños (ver Nohlen, D., 2015c) se prestan fácilmente a malentendidos cuando, por ejemplo en Levine, D.H. \& Molina, J.E (2011), se hace depender la calidad de la representación y de la integridad electoral, así como de la democracia del grado de proporcionalidad entre votos y escaños. La máxima proporcionalidad puede ser una aspiración política, el grado de proporcionalidad un aspecto analítico, es fácil de mesurar (lo que le hace atractivo para estudios cuantitativos) pero no sirve para contribuir a la determinación de la calidad democrática de las elecciones.
} 
Así, la integridad electoral, no tan dependiente de la arquitectura institucional, es una exigencia de la democracia hacia adentro. De su cumplimiento depende la democracia como tipo de régimen. Las elecciones libres y honestas constituyen el elemento definitorio clave de la democracia. Procuran la legitimidad del régimen, su reconocimiento como democracia, y procuran también la legitimidad del ejercicio del poder por parte de los ganadores electorales. Sin embargo, como estamos observando, la relación causal es también inversa. El tipo de régimen es la variable institucional decisiva para el grado de integridad electoral y el significado y la estructura de la arquitectura institucional en ella. ${ }^{17}$ América Latina, como región, constituye un buen ejemplo. En general, ha habido grandes progresos en el desarrollo de la justicia electoral. Es necesario reconocer esto, ${ }^{18}$ considerando en primer lugar que la organización de elecciones incluidas todas las etapas del ciclo electoral es el mayor procedimiento administrativo que un Estado tiene que dominar, y, segundo, teniendo en cuenta que el estado de la administración pública en general, a veces es muy deficiente. Sin embargo, en algunos países latinoamericanos, en los que cambió el régimen político hacia un autoritarismo con diferentes adjetivos, las elecciones perdieron dramáticamente su anterior carácter de ser libres y honestas (Nohlen, 2014b, Alarcon \& Casal, 2014, Nohlen, 2016b). De modo que el tipo de régimen constituye la diferencia que hace la diferencia.

\section{Variables de contexto en América Latina}

No cabe duda que hay muchos factores de contexto que influyen en la integridad electoral, y que pueden, por lo demás, jugar un rol muy

\footnotetext{
17 Cuando se cataloga a todos los países como democráticos, como procede el Proyecto de Integridad Electoral, el tipo de régimen pierde valor como factor explicativo para las normas y las prácticas electorales. En contraste, donde se diferencia entre ellos, la variable tipo de régimen puede jugar un rol importante en la explicación de la arquitectura institucional. (Lust-Okar, E. \& Ahmad, J.A., 2002).

18 Este avance está puesto de relieve también por parte de Carreras e Irepoglu (2013) cuando hablan de un "significant improvement in the quality of electoral process in the region", contrastando con el bajo grado de confianza en las elecciones. También puede consultarse Seligson, M. A. \& Maldonado, A. (2014).
} 
diferente según lugar y tiempo. O sea, no es fácil establecer generalizaciones. Hay que ser escéptico frente a cada una, incluida esta misma. Por ejemplo, en los casos, en los que el grado de integridad electoral es bajo, se llama la atención al bajo grado de desarrollo económico, o a la alta desigualdad social, o la poca experiencia con la democracia. ${ }^{19}$ Sin embargo, en comparación mundial, es bastante fácil encontrar casos que se oponen a este tipo de generalizaciones. La India es un ejemplo que contradice a menudo las tesis supuestamente universales. ${ }^{20}$ Un factor de contexto que para América Latina contiene un alto valor explicativo dentro del grupo de variables socio-culturales es el de la desconfianza generalizada hacia las instituciones.

Ella se alimenta de varios factores. Se nutre, primero, de la desconfianza social, la desconfianza en el otro que es comparativamente alta. Proviene, segundo, de la visión que la gente tiene de la política y de los políticos, en general, confirmada por el alto grado de corrupción evidenciado por hechos comprobados. En tercer lugar, se alimenta del personalismo en la política, dado que los actores que practican el personalismo a menudo se consideran por encima de la ley. Piensan con frecuencia que "el fin justifica los medios". Se nutre, cuarto, de la falta de cultura institucional, lo que se demuestra, por ejemplo, en el hecho de que la gente acepta con facilidad que el político lesione la ley cuando el objetivo le conviene, postura que corresponde al criterio y a la conducta que se practican también en las relaciones sociales en general.

Aplicando un enfoque de la teoría de juegos, Jesús Tovar Mendoza (2016) emplea el concepto de 'meta-reglas' para ilustrar los procesos electorales de mala calidad: "La primera 'meta-regla' de todo juego, incluido el electoral (un tipo específico de juego competitivo) es: el que hace trampa, gana. En ese sentido, un desnivelamiento o ventaja de alguno de los jugadores perjudica automáticamente al adversario".

\footnotetext{
19 Véase la nómina de factores causantes en Carreras, M. \& Irepoglu, Y. (2013).

20 Me refiero a diferentes tesis sobre la relación causal entre pobreza y autoritarismo, o desigualdad y autoritarismo, o extrema heterogeneidad social y autoritarismo. Ver: Sen, A. (2009) Berg-Schlosser, D. \& Kersting, N. (Eds.) (2000).
} 
"La segunda 'meta-regla' de cualquier juego es: aquel que coopta al árbitro, gana”. Tovar Mendoza (2016) desglosa diferentes maneras de lograrlo: "Uno, a través de la influencia que los actores políticos tengan para nombrar a los árbitros electorales, de tal manera que los designados tengan un vínculo de lealtad con los que decidieron su nombramiento. Dos, a través de la corrupción directa, es decir por algún tipo de beneficio económico que el acvtor político pueda otorgar directamente al juez. Tres, a través de la amenaza dirigida contra el árbitro por parte de los jugadores, ya sea sobre su actual situación laboral o incluso contra su integridad física, o sobre su futura condición profesional a través de un posible veto. "La tercera meta-regla del juego es: el que utiliza la violencia, gana. Esta es la última estrategia por la cual prima un ganador (...)". La estrategia consiste en eliminar al adversario. Se clausura el juego competitivo de la democracia, y se pasa "a un régimen autoritario, lo cual incluso conlleva a la desaparición de los árbitros, o en todo caso a que pasen a ser figuras pasivas o decorativas del nuevo sistema político." (2016, [en prensa]).

Por cierto, la desconfianza es ubicua y puede ser legítima y aconsejable. Además, conviene diferenciar entre distintos grados de desconfianza. América Latina, en general, y como confirman las encuestas, parece representar un caso de extrema desconfianza. En el campo políticoinstitucional, pone en cuestión cualquier institución o práctica política. Es interesante observar que, en general, la extrema desconfianza atañe, sobre todo, a las instituciones democráticas, las de mayor grado de equidad, incluyendo a los órganos que velan por estos valores, como los electorales. Pero la confianza en los órganos electorales supera la que consigue el poder judicial en general.

En términos generales, la desconfianza aparece como un fenómeno de resultado, de experiencias y observaciones vividas. Estas experiencias, sin embargo, inducen a los individuos y colectivos a pensar que el cumplir con el derecho y la ley resulte poco beneficioso y que, al final, solo los "tontos" terminen respetando estándares éticos. Así, la desconfianza es de igual manera un fenómeno de entrada: motiva el fraude. Genera suponer, que el otro hace fraude, e incentiva y legitima, tanto individual comocolectivamente, a un comportamiento 
igualmente deshonesto para compensar. Así se entiende, por qué los partidos luchan tanto por poner a su gente en los órganos electorales, y por qué presionan tanto a los consejeros electorales para que entren en su juego por el poder.

\section{Evaluando la evaluación de la integridad electoral}

En mis últimas consideraciones, quisiera apuntar unas observaciones generales en relación con la evaluación de la integridad electoral, en especial por parte del índice de Percepción de Integridad Electoral (PEI). ${ }^{21}$ Por cierto, el PEI es solamente una parte del proyecto, que incluye también estudios temáticos profundos. Sin embargo, el PEI parece ser el hito de la medición con más capacidad de llamar la atención. Por lo demás, es el estudio comparativo que incluye a todos los países que celebran elecciones durante los años en estudio. Esta globalidad no se alcanza hasta ahora en los demás resultados del proyecto entregados al público. ${ }^{22}$ Una excepción constituye el libro titulado Advancing Electoral Integrity que también contiene dos contribuciones sobre América Latina (Ugues, A., 2014; Seligson, M.A. \& Maldonado, A., 2014).

Una primera observación se refiere al alcance y a la manera de la evaluación. La medición incluye todas las elecciones en el mundo, no importa el tipo de régimen político. En los estudios sobre elecciones de los años setenta y ochenta, se diferenciaba entre elecciones competitivas, no-competitivas y semi-competitivas, acorde con el tipo de régimen: democrático, totalitario y autoritario. ${ }^{23}$ La categoría definía el acceso analítico. En los estudios sobre integridad electoral no exis-

\footnotetext{
21 Ver Norris, P., Martínez i Coma, F., \& Frank, R.W. (2013), y Norris, P., Frank, R. W., \& Martínez i Coma, F. (Eds.) (2014).

22 Por ejemplo, América Latina no está bien representada. Aparecen México y Cuba, pero no Bolivia, Ecuador, Nicaragua y Venezuela, para solo nombrar los países de baja o ninguna integridad electoral, más aún, son países que no pudieron mantener el estándar de integridad electoral que habían alcanzado en tiempos anteriores. Recuerdo el enorme esfuerzo de las Naciones Unidas en su misión de ONUVEN para garantizar elecciones libres y honestas en Nicaragua el año 1990, ganadas por la oposición al régimen sandinista, en las fui consultor del Jefe de misión junto a mis colegas Rafael López Pintor \& José Ignacio Wert.

23 Ver Hermet, G., Rose, R., \& Rouquié, A. (1978) ; Nohlen, D. (1978); Nohlen, D. (1981).
} 
te distinción categorial. Se determina el grado de cercanía de cada elección con el estándar internacional. La evaluación es gradual y no categorial, no se excluye ningún sistema político. Algo de integridad electoral se encuentra en cada caso. El ejemplo paradigmático es Cuba. Es una dictadura unipartidista, la oposición se encuentra oprimida o en la cárcel, las elecciones no tienen la función de representar un pluralismo político, no hay competencia ni alternancia. Sin embargo, el caso forma parte de la evaluación y consigue incluso un grado medio de integridad electoral, por encima del valor medio mundial y por encima de otros países latinoamericanos, como Ecuador, Paraguay, Venezuela y Honduras. No es solo una falla tal cual, es una falta de criterio. Cuando los resultados de la evaluación contradicen tan claramente el sentido común del público en general, se distorsiona la apreciación de todo el esfuerzo analítico. ${ }^{24}$

En segundo lugar, se desatiende el contexto sociocultural, que puede ser determinante respecto al éxito que tenga un determinado diseño institucional, aunque no corresponda al estándar internacional. El ejemplo paradigmático puede ser Alemania. Allí, el órgano electoral que organiza las elecciones es una oficina dependiente del Ministerio del Interior que no opera permanentemente, sino ad hoc, el control del financiamiento de los partidos políticos y de las campañas electorales está en manos de la presidencia del parlamento, un órgano no judicial, sino político que en caso de lesionar las normas sentencia duras sanciones a los partidos políticos; el control de los resultados electorales lo ejerce en realidad una comisión del parlamento recién elegido en función del pleno, dado que es raro que las quejas por motivo de sus sentencias lleguen al órgano judicial que en última instancia es el Tribunal Constitucional Federal. El constitucionalista Hans Meyer (1994), a quien le gusta extremar sus juicios, habla de un "escándalo de Estado de derecho", de un "procedimiento electoral totalmente separado de cualquier revisión judicial” (p. 353). ${ }^{25}$ A pesar de esta no

24 En contraste con esta extraña evaluación, Pippa Norris, Richard W. Frank \& Ferran Martínez i Coma cuentan en su libro sobre Contentious Elections (2015, p. 143), a Cuba "entre los regímenes más represivos del mundo".

25 Mis informes al respecto en Nohlen, D. (1992); Dieter Nohlen, D. (2001) 
correspondencia con los estándares internacionales, con relativamente poca inversión y cuidado en lo electoral, el electorado alemán sigue mostrando una alta confianza en los resultados electorales. ${ }^{26}$ En el PEI, Alemania aparece en el ranking de integridad electoral en uno de los primeros puestos.

Respecto a la arquitectura electoral, lo que conviene respetar es el diseño institucional en su contexto. Desde esta perspectiva, parece poco convincente abarcar la garantía de integridad electoral a partir de la arquitectura institucional misma, con un supuesto "mejor sistema" en mente. Por lo demás, debido al peso de la tradición, las reformas institucionales parecen estar sujetas a la dependencia de sendero. En vez del institucionalismo puro, debiera considerarse el estado de integridad electoral a partir del reconocimiento que reciben las elecciones como libres y honestas en términos de totalidad y honestidad, debido al examen crítico de la práctica electoral. En caso de un grado insuficiente de reconocimiento de integridad electoral aunque empíricamente comprobada, entra en juego el aspecto

26 Un cierto indicador lo constituyen las quejas electorales (Wableinsprüche) que se dirigen al Parlamento. Hubo 1453 en las elecciones de 1994, 110 en las realizadas en el año 1998; 193 en 2005, y 163 en 2013; (véase Feldkamp, 2015). El número sorprendentemente alto de 1994 se explica por el inesperado aumento en estas elecciones de los escaños excedentes a 16, doce de ellos para el partido mayoritario, que fueron determinantes en la formación de la coalición de la Democracia Cristiana con los Liberales. No se puede concluir del excepcional aumento de 1994, como lo hizo Norris, que "after 1990, rising number of petitions appealing against the results are an indication of growing suspicion by the electorate" (2015, p.134). La confianza en scholars que han detectado algo sorprendente, parece comparativamente muy alta en el Proyecto de Integridad Electoral. En el caso de Alemania unos alumnos encontraron "systematic irregularities in vote counts for Bundestag elections" (Norris, 2014, p. 5). La información se basa en una sola fuente, sin control. Tengo que limitarme aquí a decir que hay literatura especializada en alemán sobre errores electorales (Wablfebler), o sea sobre aspectos administrativos y jurisdiccionales de las elecciones, sobre todo en el derecho público y administrativo. Ver Rauber; N.D. (2005). Por otra parte, destaca la cantidad de quejas dentro del total que se refieren no al proceso electoral, sino a algunos efectos del sistema electoral de representación proporcional personalizado, a veces difíciles de entender: escaños excedentes, compensación de escaños, peso negativo del voto, etc., de modo que estas quejas se podrían también interpretar como participación ciudadana en la política. Es abundante la literatura en alemán sobre los efectos del sistema de representación proporcional personalizado a veces cuestionables que llevaron a su reforma en 2013. Sobre el rol también cuestionable del Tribunal Constitucional Federal en esto ver Nohlen, D. (2014 6b). 
institucional, considerando el probable efecto que pueda tener una reforma institucional para mejorarlo. Hay que tomar en cuenta, sin embargo, que a menudo son factores no-institucionales los que promueven un grado limitado de reconocimiento de integridad electoral en términos de legitimidad, sustanciándose en una supuesta falta de integridad electoral. Cuestionar la credibilidad electoral a veces conviene a los actores políticos. Les permite a los perdedores llamar la atención a factores fuera de su responsabilidad para explicar malos resultados y permite a los politólogos poco sensibles al contexto alimentar la idea de que una reforma institucional puede subsanar la mala práctica, conscientes de que son ellos los que manejan la sabiduría institucional, sobre todo a nivel de modelos. Cuando se trata de factores no-institucionales, es más bien una incógnita cómo puede entrar un conocimiento evaluativo de tipo institucional en el mundo de comportamiento y acción.

En tercer lugar, es importante captar bien las normas y la práctica en el ámbito administrativo y judicial, y diferenciarlas de normas políticas, o sea, de normas expuestas a legítimos criterios políticos como es el caso del sistema electoral. Esta institución define cómo los electores expresan sus preferencias políticas, es decir, cómo se vota y cómo los votos se convierten en escaños o en puestos de poder. El sistema electoral constituye las reglas que gobiernan este proceso de conversión. En América Latina, sin embargo, a menudo se aplica un concepto más amplio de sistema electoral que incluye la estructura institucional de todo el proceso electoral, desde el registro de electores hasta el contencioso electoral. En los estudios de evaluación de las elecciones, frecuentemente se mezclan todos los elementos del proceso electoral, aunque no resulta posible aplicar los mismos criterios a todos ellos. Mientras que es legítimo que el sistema electoral (en su sentido estricto) influya en el resultado electoral, es incluso una de sus funciones -y, en general, el sistema electoral favorece al partido más votado-, el resultado electoral no debe depender del reglamento administrativo y judicial. Los respectivos órganos electorales tienen que velar por la justicia electoral. Por cierto, existen maneras de manipular los sistemas electorales, y una clásica manera se refiere a la distritación, la repartición de las circunscripciones electorales -la 
respectiva manipulación lleva el nombre de gerrymandering - con alta incidencia en los sistemas de representación por mayoría, con poco efecto en el caso de sistemas de representación proporcional. Pero esta posibilidad de manipulación no legitima desatender la diferencia de sentido, función y evaluación entre el sistema electoral en su concepto amplio y el restringido.

Por otra parte, importa también que el análisis sea correcto. Por ejemplo, en la evaluación de integridad electoral por parte del PEI, el pequeño capítulo sobre Alemania contiene dos errores: el primero se refiere al sistema electoral, y el segundo a una sentencia del Tribunal Constitucional Federal. Estos errores fácticos, más no de interpretación. En el quehacer evaluativo en general, la interpretación juega un importante rol. La evaluación comparativa de alcance mundial se basa en el estudio caso por caso por parte de diferentes expertos. ${ }^{27}$ A nivel individual, importa su capacidad de análisis, su experiencia comparativa (conviene recordar que el que sólo conoce su país, no lo conoce bien) y el control de su compromiso político, a nivel colectivo, la homogeneidad de los criterios. Por cierto, influye el contexto del investigador, la persona del investigador en su contexto, guiada por intereses cognitivos, también por compromisos políticos e ideológicos que influyen en lo que observa, describe y evalúa. En este sentido, es difícil conseguir una homogeneidad de criterios del grupo de evaluadores. Es justo mencionar que los colaboradores del Proyecto de Integridad Electoral se han dado cuenta de la importancia del factor "experto en su contexto" en la evaluación, consideran que la calidad de las elecciones no es tan fácil de medir (aunque seguramente mucho más fácil que la calidad de la democracia) y de que diferencias en cuanto a conceptos, indicadores y datos llevan a resultados distintos (lo que caracteriza desde lejos los estudios cuantitativos (Levine, D.H., \& Molina, J. E., 2011; Morlino, L., 2014) sobre la calidad de la democracia) (Kelley, 2010; Van Ham, 2015).

\footnotetext{
27 Para las propias consideraciones de los autores del PEI en relación a la selección de expertos ver Ferran Martínez i Coma, F., \& Frank, R.W. (2015).
} 
La cuarta observación se refiere explícitamente a la evaluación de lo observado, reconsiderando el concepto de integridad electoral. Un ejemplo paradigmático es México. En la evaluación de las elecciones de 2012 por parte del PEI se dice: "Durante la elección hubo manifestaciones masivas en Ciudad de México en contra de supuestos sesgos en favor del PRI y de su candidato Peña Nieto en la prensa y la televisión. Después de las elecciones, (el candidato perdedor) López Obrador pidió un completo recuento de los votos, reclamando amplias irregularidades, incluidas la compra de votos (a través de tarjetas de crédito en supermercados) y el uso de fondos ilícitos por parte del PRI. El Tribunal Electoral del Poder Judicial de la Federación rechazó las acusaciones por insuficiencia de pruebas. La misión de observadores de la OEA elogió las elecciones como pacíficas y ordenadas, con administración profesional. Las protestas, sin embargo, continuaron, algunas violentas. El PEI ubicó a México en la categoría de integridad moderada, debido en parte a algunos problemas en la fase post-electoral.” (Norris, Frank, \& Martínez i Coma, (Eds.) (2014), cuadro principal).

Se desprende que en la evaluación final que se expresa en el grado de integridad electoral que se concede al caso, se integra no sólo lo observado por expertos en relación a todas las etapas del proceso electoral en términos de la totalidad, aplicando en su examen el parámetro de la relación de la práctica con los valores y normas en términos de la honestidad, sino también la percepción por parte de la opinión pública y de los actores políticos mismos, de la que se sabe que está muy influida por factores socioculturales, por la desconfianza sistémica, y precisamente por no reconocer el resultado electoral por parte del perdedor electoral. De esta manera, la estructura del concepto de integridad se acerca al de legitimidad, sustanciándose en ambos casos en dos dimensiones, una objetiva, la dignidad a la que se refieren, y una subjetiva, la creencia en la que se enfocan, como lo hemos explicado antes. Mientras que en el caso de la legitimidad, los clásicos de la teoría democrática favorecen a la creencia de legitimidad como elemento empírico clave en la evaluación politológica, a mi modo de ver, en el caso de la integridad electoral, tendría que favorecerse el examen empírico de la dignidad, o sea honestidad como parámetro 
de evaluación. Además, el cumplimiento de la integridad electoral, la honestidad demostrada y comprobada durante todo el proceso electoral, tendría que promover precisamente su activa defensa por parte de los evaluadores frente a intentos de negarla y aniquilarla. Es un servicio necesario en defensa de la democracia.

Quisiera terminar con unas preguntas y una tesis: ¿es correcto, es conveniente, unir al concepto de integridad electoral la desconfianza en ella debido a condiciones socioculturales e intereses políticos? ¿ $\mathrm{Si}$ la respuesta es sí, no se entrega a los factores socioculturales contingentes cualquier resultado del análisis evaluativo de la integridad electoral, sin poder reconocer, en su caso, el resultado objetivo de una excelente labor de los órganos electorales? Entre mayor la desconfianza en el proceso electoral como variable sociocultural en la integridad electoral y su examen en América Latina, menor el impacto que tenga la arquitectura electoral y el desempeño de los órganos electorales no solo en la integridad electoral y su evaluación, sino también en el aumento de la legitimidad de la democracia en la región.

\section{Referencias}

ACE (The Electoral Knowledge Network). (2012): Electoral Integrity (3 edición). Estocolmo: IDEA Internacional.

Alarcón, B., \& Casal, J. M. (2014). Proyecto Integridad Electoral Venezuela: las reformas impostergables, Caracas: Universidad Católica Andrés Bello.

Beber, B., \& Scacco, A. (2012) What the Nmber Say: A Digital Based Test FOR Election Fraud, Political Analysis 20(2), 211-234.

Berg-Schlosser, D., \& Kersting, N. (Eds.) (2000). Armut und Demokratie. Politische Partizipation und Interessenorganisation der städtischen Armen in Afrika und Lateinamerika. Francfort del Meno: Campus.

Birch, S. (2010) Perceptions of Electoral Fairness and Voter Turnout, Comparative Political Studies 43(12), 1601-1622.

Bobbio, N. (1981): Max Weber e Hans Kelsen. En: Max Weber e il diritto, (pp. 135-154). Milán: Franco Angeli.

Carothers, T. (1997) The Rise of Election Monitoring. The Observers Observed, Journal of Democracy 8(3), 17-31. 
Carreras, M., \& Irepoglu, Y. (2013). Perceptions of Electoral Integrity, Efficacy, and Electoral Participation in Latin America, Electoral Studies 32(4), 609-619.

Carreras, M., \& Posvar Hall, W. (2014). Who votes in Latin America? A Test of Three Theoretical Perspectives, Comparative Political Studies 47(8), 1079-1104.

Carter Center. (2013). Election Obligations and Standards. A Carter Center Assessment Manual. Atlanta: The Carter Center.

Centro de Estudios Políticos. (2014) Proyecto Integridad Electoral Venezuela. Reporte especial: Universidad Católica Andrés Bello.

Cordenillo, R. \& Ellis, A. (Eds.). (2012) The Integrity of Elections. The Role of Regional Organizations. Estocolmo: IDEA Internacional.

Darcy, R., \& McAllister, I. (1990) Ballot Position Effects, Electoral Studies 9(1), 5-17.

Easton, D. (1979). A Systems Analysis of Political Life, (3 edición). Chicago: Chicago University Press.

Elklit, J., \& Svensson, P. (1997). What Makes Elections Free and Fair?, Journal of Democracy 8(2), 32-46.

Feldkamp, M.F. (2015). Deutscher Bundestag 1994 bis 2014. Parlaments- und Wablstatistik. Baden-Baden, Nomos.

Global Commission on Elections, Democracy and Security. (2012). Deepening Democracy: A Strategy to Improve the Integrity of Elections. Estocolmo: IDEA Internacional.

Hermet, G. Rose, R. \& Rouquié, A. (Eds.) Elections without Choice. Nueva York: John Wiley \& Sons.

IDEA Internacional, Tribunal Electoral, UNAM. (2013). Justicia electoral. El manual de IDE A Internacional. México: IDEA Internacional/ Tribunal Electoral del poder Judicial de la Federación, UNAM.

IFE, Colegio de México. (2014). Informe país sobre la calidad de la ciudadanía en México. México: Instituto Federal Electoral.

Jaramillo, J. (2007). Los órganos electorales supremos. En: Nohlen, D., Zovatto, D., Orozco, J. J., Thompson, J. (Eds.). Tratado de derecho electoral comparado de América Latina (pp. 371-436). México: FCE, et. al.

Jaramillo, J., León-Roesch, M., \& Nohlen, D. (1989). Poder Electoraly consolidación democrática. Estudios sobre la organización electoral en América Latina. San José de Costa Rica: Instituto Interamericano de Derechos $\mathrm{Hu}-$ manos y Centro de Asesoría y Promoción Electoral. 
Kelley, J. (2010) Election Observers and Their Biases. Journal of Democracy $21(3), 158-172$.

Koppel, J.G.S., \& Stehen, J. A. (2004) The Effects of Ballot Position on Election Outcomes. Journal of Politics 66, 267-281.

Krennerich, M. (1992). Die Kompetitivität der Wahlen in Nicaragua, El Salvador und Guatemala in historisch vergleichender Perspektive. Ibero-Amerikanisches Archiv, Neue Folge 18(1-2), 245-290.

Leemann, L., \& Bochsler, D. (2014). A Systematic Approach to Study Electoral Fraud. Electoral Studies 35, 33-47.

Levine, D.H., \& Molina, José E. (2011). The Quality of Democracy in Latin America. Londres: Lynne y Rienner Publishers.

Lust-Okar, E., \& Amaney Ahmad, J. (2002). Rulers and Rules. Reassessing the Influence of Regime Type on Electoral Law Formation. Comparative Political Studies 35(3), 337-366.

Lusverti, C. F. (2014). Observación electoral como garantía de procesos electorales libres y justos en el marco de la Carta Democrática Interamericana. Recuperado de: http://apps.ucab.edu.ve/medialab/cuadernos/wp-content/ uploads/sites/4/2014/08/Lusverti.pdf Mandujano Rubio, S. (2006). Cooperación electoral y el compromiso internacional con la democracia. Toluca: Instituto Electoral del Estado de México.

Merloe, P. (2008) Promoting Legal Frameworks for Democratic Elections: An NDI Guide for Developing Election Laws and Law Commentaries. Washington, D.C.: National Democratic Institute for International Affairs.

Meyer, H. (1994). Der Überhang und anderes Unterhaltsame aus Anlass der Bundestagswahl 199. Kritische Vierteljahresschrift für Gesetzgebung und Rechtswissenschaft 77(4), 353.

Morlino, L. (2014). La calidad de las democracias en América Latina. Estocolmo y San José: IDEA Internacional.

Nikolayenko, O. (2015) Do Contentious Elections Depress Turnout? En P. Norris, R.W. Frank, \& F. Martínez I Coma (Eds.). Contentious Elections (pp.25-44). Nueva York y Londres: Routledge.

Nohlen, D. (1978). Wahlsysteme der Welt. Múnich: Piper.

Nohlen, D. (1981). Sistemas electorales del mundo. Madrid: Centro de Estudios Constitucionales.

Nohlen, D. (1992). La calificación electoral en Alemania. En H. Cámara de Diputados, Universidad Nacional Autónoma de México. Sistemas de calificación electoral (pp. 15-25), México: H. Cámara de Diputados/ UNAM, 15-25. 
Nohlen, D. (Ed.) (1993). Enciclopedia electoral de América Latina y del Caribe. San José de Costa Rica: Instituto Interamericano de Derechos Humanos/ Centro de Asesoría y Promoción Electoral.

Nohlen, D. (2001). Calificación electoral en Alemania Federal. En Orozco Henríquez, J. (Ed.), Sistemas de justicia electoral. Evaluación y perspectivas (pp. 61-76). México: IFE, PNUD, UNAM, IFES, IDEA International.

Nohlen, D. (2004) Sistemas electorales y partidos políticos. México: Fondo de Cultura Económica.

Nohlen, Dieter (Ed.) (2005). Elections in the Americas. Oxford: Oxford University Press.

Nohlen, D. (2006). Método comparativo. En: D. Nohlen, Dieter et al. 2006: Diccionario de Ciencia Política, México: Editorial Porrúa, segundo tomo, 871-882.

Nohlen, D. (2006). El institucionalismo contextualizado. La relevancia del contexto en el análisis y diseño institucionales. México: Porrúa y UNAM.

Nohlen, D. (2008). Justicia electoral y sus desafíos actuales en América Latina. En: D. Nohlen, Derecho y politica en su contexto. Introducción de Pedro Salazar Ugarte, (pp. 63-78). México: Suprema Corte de Justicia de la Nación.

Nohlen, D. (2010). Introducción a los sistemas electorales de los países árabes e islámicos, Elecciones 10,117-139.

Nohlen, D. (2013). Ciencia politica comparada. El enfoque histórico-empírico. Puebla y Bogotá: ICGDE y Universidad del Rosario.

Nohlen, D. (2014a). Wablrecht und Parteiensystem. Toronto: Barbara Budrich.

Nohlen, D. (2014b). Principio mayoritario y regímenes presidenciales en América Latina. Lima: Jurado Nacional de Elecciones.

Nohlen, D. (2015a). Ciencia política y justicia electoral. México: UNAM.

Nohlen, D. (2015b) Legitimität. En D. Nohlen \& F. Grotz (Eds.). Kleines Lexikon der Politike (6 edición). Múnich: C.H. Beck, 353-357.

Nohlen, D. (2015c). Gramática de los sistemas electorales. Una introducción a la ingeniería de la representación. Madrid: Tecnos.

Nohlen, D. (2016). Principio mayoritario, jurisdicción constitucional e integridad electoral en América Latina. México: UNAM.

Nohlen, D., Picado, S., \& Zovatto, D. (Comps.) (1998). Tratado de derecho electoral comparado de América Latina. México: Fondo de Cultura Económica.

Nohlen, D., Zovatto, D., Orozco, J., \& Thompson, J. (Comps.) (2007). Tratado de derecho electoral comparado de América Latina, (2 edición). México: Fondo de Cultura Económica. 
Norris, P. (2013). The New Research Agenda Studying Electoral Integrity, Electoral Studies 32(4), 563-575.

Norris, P. (2015) Why Elections Fail. Cambridge: Cambridge University Press.

Norris, P., Martínez i Coma, F. \& Frank, R. W. (2013) Assessing the Quality of Elections, Journal of Democracy 24(4), 124-135.

Norris, P., Frank, R. W., \& Martínez i Coma, F. (Eds.) (2014). The Year in Elections, 2013. The World's Flawed and Failed Contests. Sydney: Department of Government and International Relations, University of Sydney.

Norris, P., Frank, R.W., \& Martínez i Coma, F. (Eds.) (2014). Advancing Electoral Integrity, Oxford: Oxford University Press.

Norris, P., Frank, R.W., \& Martínez i Coma, F. (Eds.) (2015). Contentious Elections, Nueva York y Londres: Routledge.

Norris, P., Elklit, J. y Reynolds, A. (2014). Methods and Evidence. En: Norris, P., Frank, R.W. y Martínez i Coma, F. (Eds.) (2014), Advancing Electoral Integrity (pp. 34-50). Oxford: Oxford University Press.

Orozco Henríquez, J. (Comp.) (1999a). Justicia electoral en el umbral del siglo XXI. México: Tribunal Electoral del Poder Judicial de la Federación.

Orozco Henríquez, J. (Comp.) (1999b). Ética y derecho electoral en el umbral del siglo XXI, México: Tribunal Electoral del Poder Judicial de la Federación et al.

Ortiz Ortiz, Richard (Ed.) (2009). Estudios de justicia electoral. Quito: Tribunal Contencioso Electoral.

Pérez Duarte, J.A. (2014). El impacto de la administración electoral en la democracia latinoamericana. Toluca: Instituto Electoral del Estado de México.

Rauber, D. N. (2005). Wahlprüfung in Deutschland. Baden-Baden: Nomos.

Rodríguez Rapa, J.C., \& Seligson, M. A. (Eds.) (2012). Cultura política de la democracia en Colombia y las Américas. Bogotá: Universidad de los Andes.

Sartori, G. (1991). Comparing and Miscomparing, Journal of Theoretical Politics 3(3), 243-257.

Seligson, M. A., \& Maldonado, A. (2014). Electoral Trust in Latin America 2004-2012. En: P. Norris, R.W Frank \& F. Martínez i Coma, Advancing Electoral Integrity (pp. 229-245). Oxford: Oxford University Press.

Sen, A. (2009). The Idea of Justice. Londres: Penguin Books.

Sternberger, D. (1967). Max Webers Lehre von der Legitimität. En: W. Röhrich (Ed.), Macht und Ohnmacht des Politischen (pp. 11-126). Colonia: Kiepenheuer \& Witsch. 
Sternberger, D., \& Vogel, B. (Eds.), Nohlen, D. (Red.) (1969). Die Wabl der Parlamente und anderer Staatsorgane. 2 tomos. Berlín: de Gruyter.

Tovar Mendoza, J. (2016). Ensayo introductorio. En: D. Nohlen (2015), México y el desarrollo de la democracia en América Latina. Tres conferencias. México: Tribunal Electoral del Poder Judicial de la Federación.

Ugues, JR. A.(2014). Electoral Management Bodies in Central America. En: Norris, P., Frank, R.W. \& Martínez i Coma, F. (Eds.) Advancing Electoral Integrity (pp. 118-134). Oxford: Oxford University Press.

United Nations. (2005). The Declaration of Principles for International Election Observation and Code of Conduct for International Election Observers. Nueva York: United Nations Organization.

Van Ham, C. (2015). Getting Elections Right? Measuring Electoral Integrity, Democratization 22(4), 714-734.

Weber, M. (1974). Los tres tipos puros de dominación legítima, En M. Weber, Economía y sociedad (pp. 706-716). México: Fondo de Cultura Económica.

Weber, M. (2001). Ensayos sobre metodología sociológica (6 edición). Buenos Aires: Amorrortu.

Zovatto, D. (Coord.) (2006). Regulación jurídica de los partidos politicos en América Latina. México: UNAM, IDEA Internacional. 
